\title{
Combined mismatch repair and POLE/POLD1 defects explain unresolved suspected Lynch syndrome cancers
}

\author{
Anne ML Jansen ${ }^{1,2}$, Tom van Wezel ${ }^{1}$, Brendy EWM van den Akker ${ }^{1}$, Marina Ventayol Garcia ${ }^{1}$, Dina Ruano ${ }^{1}$, \\ Carli MJ Tops ${ }^{3}$, Anja Wagner ${ }^{4}$, Tom GW Letteboer ${ }^{5}$, Encarna B Gómez-García ${ }^{6}$, Peter Devilee ${ }^{2}$, \\ Juul T Wijnen ${ }^{2,3}$, Frederik J Hes ${ }^{3}$ and Hans Morreau ${ }^{\star 1}$
}

\begin{abstract}
Many suspected Lynch Syndrome (sLS) patients who lack mismatch repair (MMR) germline gene variants and MLH1 or MSH2 hypermethylation are currently explained by somatic MMR gene variants or, occasionally, by germline POLE variants. To further investigate unexplained sLS patients, we analyzed leukocyte and tumor DNA of 62 sLS patients using gene panel sequencing including the POLE, POLD1 and MMR genes. Forty tumors showed either one, two or more somatic MMR variants predicted to affect function. Nine sLS tumors showed a likely ultramutated phenotype and were found to carry germline $(n=2)$ or somatic variants $(n=7)$ in the POLE/POLD1 exonuclease domain (EDM). Six of these POLE/POLD1-EDM mutated tumors also carried somatic MMR variants. Our findings suggest that faulty proofreading may result in loss of MMR and thereby in microsatellite instability.
\end{abstract}

European Journal of Human Genetics (2016) 24, 1089-1092; doi:10.1038/ejhg.2015.252; published online 9 December 2015

\section{INTRODUCTION}

Inactivation of the mismatch repair (MMR) genes $M L H 1, M S H 2$, MSH6 and PMS2 causes Lynch syndrome (LS), an autosomal dominant predisposition for colorectal and endometrial cancer. ${ }^{1}$ Inactivation of the mismatch repair pathway can also occur sporadically, through somatic $\mathrm{MLH1}$ hypermethylation ${ }^{1}$ or by acquired biallelic somatic inactivation (variant affecting function or loss of heterozygosity (LOH)) of the MMR genes. ${ }^{2,3}$ Inaccurate DNA repair leads to a high frequency of somatic variants, with loss of MMR leading to 'hypermutated' tumors with $10-100$ variants/Mb. ${ }^{4}$ LS tumors are characterized by microsatellite instability (MSI) and immunohistochemical loss of expression of MMR proteins. ${ }^{1}$ However, germline variants affecting function cannot be detected in up to $59 \%$ of patients displaying MSI and/or loss of MMR, referred to as 'suspected LS' (sLS)..$^{5}$

Recently, germline and somatic variants in the exonuclease domains (EDMs) of DNA polymerase $\varepsilon$ (POLE) and polymerase d (POLD1) were described. ${ }^{6-14}$ These POLE/POLD1 variants affect proofreading function and lead to an ultramutated phenotype with a variant incidence exceeding 100 variants/Mb. Germline POLE-EDM variants can result in a LS phenotype and microsatellite instable colorectal cancers (CRCs). ${ }^{6,15}$ The exact role of somatic POLE/POLD1 variants in tumors with high microsatellite instability (MSI-H) remains unclear.

The aim of our study was to identify the underlying genetic cause of disease in a cohort of 64 sLS cases - selected on the basis of MSI, loss of MMR, young onset and often a family history for LS - by screening the MMR, POLE and POLD1 genes in both leukocyte and tumor DNA.

\section{SUBJECTS AND METHODS}

This study included 64 patients with Lynch-associated tumors recruited in four academic centers in the Netherlands between 1997 and 2014: Leiden University Medical Centre $(n=37)$, Maastricht University Medical Centre $(n=11)$, Erasmus Medical Centre $(n=9)$ and University Medical Centre Utrecht $(n=7)$. Demographic and clinical data, as well as informed consent, were obtained at the time of diagnosis. This study was approved by the local medical ethical committee of the LUMC (P01-019E). Patients were selected based on loss of MMR (as indicated by immunohistochemical staining) and/or MSI. Unexplained tumors with low MSI or tumors with inconclusive IHC results were also included in this study (see Supplementary Table 1 and Supplementary Methods).

Fifty-eight (91\%) patients fulfilled Bethesda criteria, ${ }^{16}$ and families of 24 (38\%) patients also fulfilled Amsterdam II criteria. ${ }^{17}$ Patients were previously screened in a diagnostic setting for germline MMR variants. Whereas 57 patients showed no disease-causing germline variants, 7 patients were found to have a germline variant of unknown significance (VUS). Of the total cohort, $75 \%$ of patients presented with CRC $(n=48), 14 \%$ with endometrial cancer $(\mathrm{EC}, n=9)$ and $11 \%$ with another LS-associated tumor (see Supplementary Table 1).

The average age of onset was 52.1 years. Two patients were excluded from the analysis because of poor DNA quality. Of the remaining 62 tumors, tumor and leukocyte DNA was sequenced for variants in the exonic regions of $M L H 1$, MSH2, MSH6, PMS2, POLE and POLD1 using the Ion PGM System (Life Technologies, Carlsbad, CA, USA). Raw data analysis, alignments and variant calling was carried out using the default parameters in Torrent Suite v4.0 (Thermo Fisher Scientific, Waltham, MA, USA; see Supplementary Methods). Variants were functionally annotated using ANNOVAR. ${ }^{18}$ The full data set was filtered and prioritized by variant frequency $(>10 \%)$ and coverage $(>50 \times)$. Interesting variants under $10 \%$ were manually curated. The in silico prediction programs were used to predict effect on function (see Supplementary Methods). All variants (likely) affecting function, including two variants with a 9\% variant frequency, were validated with Sanger sequencing. For all PMS2

${ }^{1}$ Department of Pathology, Leiden University Medical Centre, Leiden, The Netherlands; ${ }^{2}$ Department of Human Genetics, Leiden University Medical Centre, Leiden, The Netherlands; ${ }^{3}$ Department of Clinical Genetics, Leiden University Medical Centre, Leiden, The Netherlands; ${ }^{4}$ Department of Clinical Genetics, Erasmus University Medical Centre, Rotterdam, The Netherlands; ${ }^{5}$ Department of Medical Genetics, University Medical Centre Utrecht, Utrecht, The Netherlands; ${ }^{6}$ Department of Clinical Genetics, University Hospital Maastricht, Maastricht, The Netherlands

*Correspondence: Professor H Morreau, Department of Pathology, Leiden University Medical Center, Albinusdreef 2, 2333 ZA Leiden, The Netherlands. Tel: +31 715266630 ; Fax: +31 71526 8285; E-mail: j.morreau@lumc.nl

Received 26 June 2015; revised 14 October 2015; accepted 14 October 2015; published online 9 December 2015 
variants, PMS2-specific primers were created to confirm that the variant is present in PMS2 and not in a PMS2 pseudogene.

LOH was determined for every heterozygous SNP by comparing the ratio of allele A with allele B in leukocyte and tumor DNA samples. Furthermore, for every heterozygous SNP, the allelic imbalance factor (AIF) ${ }^{19}$ was calculated and Fisher's exact test was performed to determine whether the difference between normal and tumor is significant. If all heterozygous SNPs of one gene showed LOH with an AIF $>2$ and Fisher's exact $P$-value $<0.05, \mathrm{LOH}$ was called (Supplementary Table 1).

\section{RESULTS}

Seven patients with a germline MMR VUS (class 3) were included in this study (see Supplementary Table 1). In all cases, the variant was detected with NGS in leukocyte and tumor DNA. During the course of the study, three of these germline variants were reclassified as class 4 or 5 ((probably) affects function) by the International Society for Gastrointestinal Hereditary Tumors Incorporated (InSiGHT). Four tumors displayed a second somatic MMR variant predicted to affect function. One tumor displayed LOH (Supplementary Table 1). Six cases with somatic MLH1 hypermethylation fulfilling revised Bethesda criteria (three from families fulfilling Amsterdam II criteria) were sequenced for underlying hereditary defects explaining the family history, but no germline variants were found. One of these MLH1 hypermethylated tumors carried a somatic $M L H 1$ variant likely to affect function and one displayed MLH1 LOH (Supplementary Table 1).

One $(n=27,44 \%)$ or two $(n=13,21 \%)$ somatic aberrations (variant or $\mathrm{LOH}$ ) in a MMR gene were found in a total of 62 tumors (see Supplementary Table 1). Biallelic inactivation was concordant with IHC. Of the 13 tumors with two somatic aberrations, 12 had variants in MLH1 or MSH2 and were MSI-H. The thirteenth tumor, sLS-07, showed expression loss of MSH6 and was MSS.

Although the majority $(83 \%)$ of tumors showed $<10$ somatic variants in the genomic region analyzed, 10 cases displayed a larger number of somatic variants, ranging from 16 to 375 somatic variants within the sequenced area of $31 \mathrm{~kb}$. Out of 10 tumors, 9 showed a POLE or POLD1 variant that (probably) affects function (Table 1). Of the highly mutated tumors, two carried novel germline heterozygous $P O L E / P O L D 1$ variants that are predicted to affect proofreading (Supplementary Table 2). Of these two germline cases, tumor sLS-67 was also found to carry two somatic MLH1 variants, explaining the tumor phenotype (loss of MLH1 and PMS2 expression and MSI-H). The second tumor, sLS-16, was MSI-L, showed positive MLH1 and MSH2 staining (MSH6 and PMS2 were not tested) and had no somatic MMR variants.

Seven of the highly mutated tumors showed somatic POLE/POLD1 variants likely to affect function. Six tumors carried a somatic POLE/POLD1-EDM hot spot variant (POLE: c.857C $>\mathrm{G}, \mathrm{c} .856 \mathrm{C}>\mathrm{T}$, c. $1231 \mathrm{G}>\mathrm{T}$, c. $1366 \mathrm{G}>\mathrm{C}, \mathrm{c} .1367 \mathrm{C}>\mathrm{T}$ or $\mathrm{c} .1376 \mathrm{C}>\mathrm{T}$ and $P O L D 1$ c. $1433 \mathrm{G}>\mathrm{A}$ ) that has previously been described to impair proofreading. ${ }^{4}$ In the seventh tumor (sLS-105), a novel POLE c.846_847delinsTT variant was detected. This variant lies close to a known POLE hot spot site (POLE c.857) and is predicted to affect function by two out of three prediction programs (Supplementary Table 2). All POLE variants were heterozygous, in agreement with previous research. ${ }^{7,9,10,13,15}$ Four POLE/POLD1-EDM mutated tumors displayed additional somatic nonsense POLE variants outside the exonuclease domain (see Supplementary Table 3). Only one of these was upstream of the exonuclease domain (sLS-16).

Eight of the nine ultramutated tumors with a POLE/POLD1-EDM variant found in our study showed MSI (3 MSI-H and 5 MSI-L). In six of these ultramutated tumors, IHC detected loss of at least one of the MMR proteins and all six tumors displayed somatic variants in the affected MMR gene likely to affect function. Tumor sLS-19 with two $P O L E$ variants was found to have two somatic $M L H 1$ aberrations, as well as two somatic PMS2 aberrations, and IHC showed solitary loss of PMS2 expression. Reanalysis of staining also showed ambiguous MLH1 staining (cytoplasmic enhancement and vague, focal nuclear staining). Tumor sLS-09 displayed a nonsense MSH6 variant and missense $\mathrm{MSH} 2$ variant predicted to affect function, and IHC showed loss of MSH6 expression and weak positive MSH2 expression. In three POLE/POLD1-EDM mutated tumors with positive or inconclusive MMR expression and MSI-L phenotype, no somatic MMR variants (likely) to affect function were found. However, in one of those three tumors (sLS-05), solitary MLH1 LOH and PMS2 LOH without variants was found (see Table 1).

In three non-ultramutated tumors, a POLE/POLD1-EDM variant was found (sLS-80, sLS-87 and sLS-101, see Table 1). These variants have not been described before, but are predicted to affect function (see Supplementary Table 2). Two variants co-occur with a germline MSH6 VUS and a somatic MSH6 variant (sLS-80 and sLS-87), whereas one (sLS-101) co-occurs with a somatic PMS2 variant. Four additional non-ultramutated tumors showed POLE (sLS-18, sLS-21) or POLD1 LOH (sLS-12, sLS-49) in all heterozygous SNPs (Supplementary Table 1), without germline or somatic POLE variants.

\section{DISCUSSION}

POLE-EDM variants are reported to be the mutagenic factor driving ultramutation in tumors. ${ }^{20}$ The same report also noted that the increased mutational load seen in POLE mutated tumors exceeds that expected because of loss of exonuclease activity. ${ }^{20}$ The number of variants detected in the sequenced area in the present study implicates an ultramutated phenotype, with $>100$ variants/Mb in all POLE/POLD1-EDM mutated tumors in this cohort. As only a limited region $(31 \mathrm{~kb})$ was sequenced, we can only extrapolate the total number of variants per $\mathrm{Mb}$. In our cohort, the POLE/POLD1 mutated MMR-deficient tumors display two deficient pathways increasing the mutational load. Comparing frequencies of the different variants found in these tumors, it might be concluded that faulty proofreading may be the initiating event in some of these tumors, possibly resulting in loss of MMR and thereby in MSI. Interestingly, four tumors show POLE/POLD1 LOH without germline or somatic POLE/POLD1 variants. These tumors however do not show the typical ultramutated phenotype, whereas single variants without $\mathrm{LOH}$ do show that. This phenomenon of $\mathrm{LOH}$ without variants affecting the exonuclease domain has not yet been described. Possibly, the remaining allele is enough to maintain proofreading. Furthermore, three tumors show somatic POLE/POLD1 variants, without the ultramutated phenotype. All three variants are missense, but are predicted to affect function (Supplementary Table 2). As these variants are not found in ultramutated tumors, evidence of deleterious functional effect is lacking.

In conclusion, targeted next-generation sequencing of 62 sLS cases led to the detection of 9 highly mutated tumors with a germline $(n=2)$ or somatic $(n=7)$ POLE/POLD1-EDM variant. Even though $P O L E$ germline variants have previously been shown to co-occur with somatic MMR variants, ${ }^{11}$ in this study we found germline and somatic POLE/POLD1 variants in a cohort selected for sLS characteristics. Importantly, although current literature mainly addresses POLE/POLD1 variants in MSS tumors, somatic POLE/POLD1 variants in sLS patients are likely to be overlooked. However, a very recent recommendation for genetic testing and surveillance states that MMR deficiency should not be an exclusion criterion for genetic testing of 
Table 1 Overview of patients with a POLE/POLD1-EDM mutated tumor

\begin{tabular}{|c|c|c|c|c|c|c|c|c|c|}
\hline Patient & $\begin{array}{l}\text { IHC-negative } \\
\text { staining }\end{array}$ & MSI & $\begin{array}{l}\text { Age of } \\
\text { onset }\end{array}$ & $\begin{array}{l}\text { Family } \\
\text { history }\end{array}$ & $\begin{array}{c}\text { Number of } \\
\text { variants }\end{array}$ & Gene & Variant & $\begin{array}{l}\text { Amino acid } \\
\text { alteration }\end{array}$ & $\%$ \\
\hline \multicolumn{10}{|c|}{ Somatic POLE/POLD1-EDM variant } \\
\hline sLS-07 & MSH6 (1) & S & 39 & TDR & 37 & $\begin{array}{l}\text { POLE } \\
\text { MSH6 } \\
\text { MSH6 }\end{array}$ & $\begin{array}{l}\text { c. } 1231 G>T \\
\text { c. } 2735 G>A \\
\text { c. } 2876 G>A\end{array}$ & $\begin{array}{l}\text { p.(V411L) } \\
\text { p.(W912*) } \\
\text { p.(R959H) }\end{array}$ & $\begin{array}{l}44 \% \\
10 \% \\
14 \%\end{array}$ \\
\hline sLS-09 & MSH6 (2) & L & 42 & FDR & 16 & $\begin{array}{l}\text { POLE } \\
\text { MSH6 } \\
\text { MSH2 }\end{array}$ & $\begin{array}{l}\text { c. } 857 C>G \\
\text { c. } 2539 G>T \\
\text { c. } 1166 G>A\end{array}$ & $\begin{array}{l}\text { p.(P286R) } \\
\text { p.(E847*) } \\
\text { P.(R389Q) }\end{array}$ & $\begin{array}{l}38 \% \\
36 \% \\
38 \%\end{array}$ \\
\hline sLS-19 & PMS2 & $\mathrm{H}$ & 45 & FDR & 221 & $\begin{array}{l}\text { POLE } \\
\text { POLE } \\
M L H 1 \\
\text { MLH1 } \\
\text { PMS2 } \\
\text { PMS2 }\end{array}$ & $\begin{array}{c}\text { c. } 1376 \mathrm{C}>\mathrm{T} \\
\mathrm{c} .856 \mathrm{C}>\mathrm{T} \\
\mathrm{c} .199 \mathrm{G}>\mathrm{A} \\
\mathrm{LOH} \\
\text { c. } 308 \mathrm{C}>\mathrm{T} \\
\mathrm{LOH}\end{array}$ & $\begin{array}{l}\text { p.(S459F) } \\
\text { p.(P286S) } \\
\text { p.(G67R) }\end{array}$ & $\begin{array}{r}21 \% \\
9 \% \\
19 \% \\
11 \%\end{array}$ \\
\hline sLS-24 & None & L & 34 & FDR & 115 & POLE & c. $1366 \mathrm{G}>\mathrm{C}$ & p.(A456P) & $28 \%$ \\
\hline sLS-66 & MSH6 & L & 66 & SDR & 25 & $\begin{array}{l}\text { POLD1 } \\
\text { MSH6 }\end{array}$ & $\begin{array}{c}\text { c. } 1433 G>A \\
\text { c.3600_3601del }\end{array}$ & $\begin{array}{c}\text { p.(S478N) } \\
\text { p.(L1201Hfs*13) }\end{array}$ & $\begin{array}{l}32 \% \\
28 \%\end{array}$ \\
\hline sLS-80 & MSH2/MSH6 & $\mathrm{H}$ & 52 & FDR & 5 & $\begin{array}{l}\text { POLD1 } \\
\text { MSH6 } \\
\text { MSH6 }\end{array}$ & $\begin{array}{c}\text { c. } 1429 \mathrm{G}>A \\
\text { c. } 3961 \mathrm{~A}>\mathrm{G}^{* *} \\
\text { c. } 3186 \mathrm{C}>\mathrm{A}\end{array}$ & $\begin{array}{l}\text { p.(V477M) } \\
\text { p.(R1321G) } \\
\text { p.(C1062*) }\end{array}$ & $\begin{array}{l}26 \% \\
52 \% \\
25 \%\end{array}$ \\
\hline sLS-87 & MSH2/MSH6 & $\mathrm{H}$ & 49 & FDR & 9 & $\begin{array}{l}\text { POLE } \\
\text { MSH6 } \\
\text { MSH6 }\end{array}$ & $\begin{array}{c}\text { c. } 1218 \mathrm{C}>\mathrm{G} \\
\text { c.3473_3475del } \text { l* }^{* *} \\
\text { c.3311_3312delTT }\end{array}$ & $\begin{array}{c}\text { p.(N406E) } \\
\text { p.(C1158del) } \\
\text { p.(F1104Trpfs*3) }\end{array}$ & $\begin{array}{l}16 \% \\
53 \% \\
21 \%\end{array}$ \\
\hline sLS-101 & PMS2 & $\mathrm{H}$ & 55 & FDR & 5 & $\begin{array}{l}\text { POLD1 } \\
\text { PMS2 }\end{array}$ & $\begin{array}{l}\text { c. } 1003 A>G \\
\text { c. } 1687 C>T\end{array}$ & $\begin{array}{l}\text { p.(I335V) } \\
\text { p. }(\text { R563*) }\end{array}$ & $\begin{array}{l}22 \% \\
30 \%\end{array}$ \\
\hline sLS-105 & MLH1/ PMS2 & $\mathrm{H}$ & 49 & No & 184 & $\begin{array}{l}\text { POLE } \\
\text { MLH1 }\end{array}$ & $\begin{array}{c}\text { c.846_847delins TT } \\
\text { c. } 1614 \mathrm{G}>\mathrm{A}\end{array}$ & $\begin{array}{l}\text { p.(L283F) } \\
\text { p. }(\text { W538*) }\end{array}$ & $\begin{array}{l}13 \% \\
13 \%\end{array}$ \\
\hline
\end{tabular}

Abbreviation: EDM, exonuclease domain.

IHC was performed for MLH1, MSH2, MSH6 and PMS2. Numbers behind staining results indicate: (1) at least MSH6 negative, (2) PMS2 not tested and (3) inconclusive testing. MSI status is defined as MSI-H (H), MSI-L (L) or MSS (S). Age of onset is the age at which the first Lynch-associated tumor occurred. All patients presented with colorectal cancer, except patients sLS-87 and sLS-101 who presented with endometrial cancer. Number of variants depicts the number of somatic variants with a frequency $>10 \%$ identified in the sequenced region of $31 \mathrm{~kb}$. Stop codons are indicated with an asterisk $\left(^{*}\right)$. Germline variants of unknown significance (VUS) are indicated with a double asterisk $\left({ }^{* *}\right)$. \% shows the percentage of variant reads. Family history is defined as a first-degree relative with LS-associated tumors (FDR), second-degree relative with LS-associated tumors (SDR), third-degree relative with LS-associated tumors (TDR) or no LS in the family (No).

germline POLE/POLD1 variants. ${ }^{21}$ Our results further emphasize the importance of POLE/POLD1 germline and somatic screening in unexplained MSI-H and MMR-deficient tumors.

\section{CONFLICT OF INTEREST}

The authors declare no conflict of interest.

\section{ACKNOWLEDGEMENTS}

This work was supported by the Dutch Cancer Society under study number UL2012-5542.

\section{AUTHOR CONTRIBUTIONS}

AMLJ: experimental work, data analysis and writing; TvW: study concept and design, supervision data analysis and variant calling, critical revision of the manuscript; BEWMvdA and MVG: experimental work; DR: bioinformatic support; CMJT and PD: patient selection; CMJT, AW, TGWL and EBGG: material collection; JTW, FJH and HM: supervision, study concept and design, critical revision of the manuscript and obtained funding.

1 Colas C, Coulet F, Svrcek M et al: Lynch or not Lynch? Is that always a question? Adv Cancer Res 2012; 113: 121-166.

2 Mensenkamp AR, Vogelaar IP, van Zelst-Stams WA et al: Somatic mutations in MLH1 and $\mathrm{MSH} 2$ are a frequent cause of mismatch-repair deficiency in Lynch syndromelike tumors. Gastroenterology 2014; 146: 643-646 e8.

3 Geurts-Giele WR, Leenen $\mathrm{CH}$, Dubbink HJ et al: Somatic aberrations of mismatch repair genes as a cause of microsatellite-unstable cancers. J Pathol 2014; 234: 548-559.

4 Shinbrot E, Henninger EE, Weinhold N et al: Exonuclease mutations in DNA polymerase epsilon reveal replication strand specific mutation patterns and human origins of replication. Genome Res 2014; 24: 1740-1750.

5 Buchanan DD, Rosty C, Clendenning M, Spurdle AB, Win AK: Clinical problems of colorectal cancer and endometrial cancer cases with unknown cause of tumor mismatch repair deficiency (suspected Lynch syndrome). App/ Clin Genet 2014; 7: 183-193. 
6 Heitzer E, Tomlinson I: Replicative DNA polymerase mutations in cancer. Curr Opin Genet Dev 2014; 24: 107-113.

7 Palles C, Cazier JB, Howarth KM et al: Germline mutations affecting the proofreading domains of POLE and POLD1 predispose to colorectal adenomas and carcinomas. Nat Genet 2013; 45: 136-144.

8 Valle L, Hernandez-IIlan E, Bellido F et al: New insights into POLE and POLD1 germline mutations in familial colorectal cancer and polyposis. Hum Mol Genet 2014; 23: 3506-3512.

9 Briggs S, Tomlinson I: Germline and somatic polymerase epsilon and delta mutations define a new class of hypermutated colorectal and endometrial cancers. J Pathol 2013; 230: 148-153

10 Spier I, Holzapfel S, Altmuller J et al: Frequency and phenotypic spectrum of germline mutations in POLE and seven other polymerase genes in 266 patients with colorectal adenomas and carcinomas. Int J Cancer 2015; 137: 320-331.

11 Haraldsdottir S, Hampel H, Tomsic J et al: Colon and endometrial cancers with mismatch repair deficiency can arise from somatic, rather than germline, mutations. Gastroenterology 2014; 147: 1308-1316 e1.

12 Yoshida R, Miyashita $\mathrm{K}$, Inoue $\mathrm{M}$ et al: Concurrent genetic alterations in DNA polymerase proofreading and mismatch repair in human colorectal cancer. Eur J Hum Genet 2011; 19: 320-325.

13 Church DN, Briggs SE, Palles C et al: DNA polymerase epsilon and delta exonuclease domain mutations in endometrial cancer. Hum Mol Genet 2013; 22: 2820-2828.

14 Billingsley CC, Cohn DE, Mutch DG, Stephens JA, Suarez AA, Goodfellow PJ: Polymerase varepsilon (POLE) mutations in endometrial cancer: clinical outcomes and implications for Lynch syndrome testing. Cancer 2015; 121: 386-394.

15 Elsayed FA, Kets CM, Ruano D et al: Germline variants in POLE are associated with early onset mismatch repair deficient colorectal cancer. Eur J Hum Genet 2014; 23 : 1080-1084.

16 Umar A: Revised Bethesda guidelines for hereditary nonpolyposis colorectal cancer (Lynch Syndrome) and microsatellite instability. J Nat/ Cancer Inst 2004; 96: 261-268.
17 Park J-G, Vasen HF, Park Y et al: Suspected HNPCC and Amsterdam criteria II: evaluation of mutation detection rate, an international collaborative study. Int JColorectal Dis 2001; 17: 109-114.

18 Wang $\mathrm{K}$, Li M, Hakonarson $\mathrm{H}$ : ANNOVAR: functional annotation of genetic variants from high-throughput sequencing data. Nucleic Acids Res 2010; 38: e164.

19 Devilee P, Cleton-Jansen AM, Cornelisse CJ: Ever since Knudson. Trends Genet 2001; 17: 569-573.

20 Helleday T, Eshtad S, Nik-Zainal S: Mechanisms underlying mutational signatures in human cancers. Nat Rev Genet 2014; 15: 585-598.

21 Bellido F, Pineda M, Aiza G et al: POLE and POLD1 mutations in 529 kindred with familial colorectal cancer and/or polyposis: review of reported cases and recommendations for genetic testing and surveillance. Genet Med 2015; e-pub ahead of print 2 July 2015; doi:10.1038/gim.2015.75.

This work is licensed under a Creative Commons Attribution-NonCommercial-NoDerivs 4.0 International License. The images or other third party material in this article are included in the article's Creative Commons license, unless indicated otherwise in the credit line; if the material is not included under the Creative Commons license, users will need to obtain permission from the license holder to reproduce the material. To view a copy of this license, visit http://creativecommons.org/licenses/ by-nc-nd/4.0/

Supplementary Information accompanies this paper on European Journal of Human Genetics website (http://www.nature.com/ejhg) 